\title{
Need for further clarity on optimal approach to ectopic canines
}

\author{
Abstracted from \\ Armi P, Cozza P, Baccetti T. \\ Effect of RME and headgear treatment on the eruption of palatally displaced canines: \\ a randomized clinical study. Angle Orthod 2011; 81: 370-374. Epub 2011 Feb 7. \\ Address for correspondence: Dr Tiziano Baccetti, Department of Orthodontics, \\ The University of Florence, Florence, Italy. E-mail: tbaccetti@unifi.it
}

\section{Question: In patients with palatally displaced maxillary canines (PDCs) is cervical pull headgear (HG) or rapid maxillary expansion and cervical pull headgear (RME/HG) more effective than no treatment in increasing the rates of eruption?}

Design Randomised controlled trial.

Intervention Patients between 8-13 years of age with either unilateral or bilateral PDC diagnosed on radiograph with the presence of mild crowding at the maxillary arch and/or molar relation showing Class II tendency were included. The head gear group (HG) used cervical pull headgear alone for one year for 12-14 hours a day, while the rapid maxillary expansion/headgear $(\mathrm{RME} / \mathrm{HG})$ group were treated with a banded rapid maxillary expander, (7 $\mathrm{mm}$ of active expansion; at the end of expansion all patients retained the expander for six months), followed by use of a cervical pull headgear as in the HG group. A control group received no active treatment.

Outcome measure A successful outcome for PDC was defined as the full eruption of the tooth, thus permitting bracket positioning for final arch alignment at 18 months after the initial observation.

Results Sixty of the 64 patients who entered the trial were available for final assessment. Successful eruption was $85.7 \%$ in the RME/HG group, $82.3 \%$ in the HG group and $36 \%$ in the control group. There was no statistically significant difference between the $\mathrm{HG}$ and RME/HG groups. Conclusions The use of rapid maxillary expansion and headgear (or headgear alone) in PDC cases increases the success rate of eruption of the canine significantly (almost three times more than in untreated controls).

\section{Commentary}

Seminal research by Ericson and $\mathrm{Kurol}^{1}$ indicated that removal of primary maxillary canines is a predictable and relatively conservative solution to the ectopically developing palatal maxillary canine. Recently, however, the merit of removing primary canines has been questioned $^{2,3}$. In a previous study by Baccetti et al., ${ }^{4}$ an alternative involving use of cervical pull headgear $(\mathrm{CHG})$ without recourse to removal of primary canines was also explored.

This randomised controlled trial considers the merits of another novel approach to the management of ectopically developing canines. Eruption rates are compared over an 18-month period following either combined use of CHG and rapid maxillary expansion (RME), CHG in isolation, or observation only in a group of 60 subjects.
The results indicate that either $\mathrm{CHG}$ in isolation or combined with RME significantly increase the rate of successful eruption compared to an untreated control. However, little difference in eruption rates was found with the addition of RME compared to use of CHG in isolation (85.7\% vs. $82.3 \%)$. The authors propose that the success of $\mathrm{CHG}$ is based on inhibition of mesial movement of maxillary first permanent molars, preserving space for eruption of the permanent canines.

This study is an interesting addition to the evidence base and appraises relatively novel approaches to interception of a significant problem. However, the inclusion of subjects as young as eight years is contentious; Ericson and $\mathrm{Kurol}^{1}$ considered subjects ranging in age from 10 to 13 years. As improvement in canine position may occur naturally during childhood, active intervention to intercept suspected canine ectopia prior to 10 years may often be unnecessary.

Limitations with the reporting of the study includes failure to justify the sample size, and inadequate explanation of randomisation procedures, allocation concealment and efforts to reduce measurement bias. Additionally, clustering effects associated with multiple observations within the same patients were not considered, risking overestimation of treatment effects. Consequently, in future systematic reviews concerning interception of ectopically developing canines, this study is likely to be overlooked. While this study is both interesting and original, further robust research to clarify the optimal approach to intercept the ectopic maxillary canine remains necessary.

\section{Practice points}

- The optimal approach to interception of the ectopic maxillary canine is unclear

- Use of cervical pull headgear may increase the likelihood of successful eruption, although further research is required to confirm this.

Padhraig S. Fleming

Consultant Orthodontist, East Kent Hospitals University NHS Foundation Trust, Canterbury, Kent, UK

1. Ericson S, Kurol J. Early treatment of palatally erupting maxillary canines by extraction of the primary canines. Eur / Orthod 1988; 10: 283-295.

2. Parkin N, Benson PE, Shah A. Extraction of primary (baby) teeth for unerupted palatally displaced permanent canine teeth in children. Cochrane Database Syst Rev 2009; 2: CD004621.

3. Naoumova |, Kurol I, Kjellberg $\mathrm{H}$. A systematic review of the interceptive treatment of palatally displaced maxillary canines. Eur J Orthod 2011; 33: 143-149.

4. Baccetti T, Leonardi M, Armi P. A randomized clinical study of two interceptive approaches to palatally displaced canines. Eur J Orthod 2008; 30: 381-385.

Evidence-Based Dentistry (2012) 13, 81. doi:10.1038/sj.ebd.6400876 